\title{
The prevention and regression of atherosclerotic plaques: emerging treatments
}

\author{
This article was published in the following Dove Press journal: \\ Vascular Health and Risk Management \\ 24 September 2012 \\ Number of times this article has been viewed
}

\author{
Atul Ashok Kalanuria' \\ Paul Nyquist ${ }^{1}$ \\ Geoffrey Ling ${ }^{1,2}$ \\ 'Division of Neuro Critical Care, \\ Department of Anesthesiology \\ and Critical Care Medicine, The \\ Johns Hopkins University School \\ of Medicine, Baltimore, ${ }^{2}$ Department \\ of Neurology, Uniformed Services \\ University of the Health Sciences, \\ Bethesda, MD, USA
}

\begin{abstract}
Occlusive vascular diseases, such as sudden coronary syndromes, stroke, and peripheral arterial disease, are a huge burden on the health care systems of developed and developing countries. Tremendous advances have been made over the last few decades in the diagnosis and treatment of atherosclerotic diseases. Intravascular ultrasound has been able to provide detailed information of plaque anatomy and has been used in several studies to assess outcomes. The presence of atherosclerosis disrupts the normal protective mechanism provided by the endothelium and this mechanism has been implicated in the pathophysiology of coronary artery disease and stroke. Efforts are being put into the prevention of atherosclerosis, which has been shown to begin in childhood. This paper reviews the pathophysiology of atherosclerosis and discusses the current options available for the prevention and reversal of plaque formation.

Keywords: cardiovascular, atherosclerotic disease, endothelium, plaque, reversal, coronary artery disease, stroke
\end{abstract}

\section{Introduction}

According to the 2012 American Heart Association (AHA) statistical update, every year, approximately 795,000 people experience a new or recurrent stroke. Stroke accounts for about one of every 18 deaths in the USA, with a stroke occurring every 40 seconds, and is a major cause of disability in the elderly. ${ }^{1}$ Although death rates from cardiovascular diseases (CVDs) have declined, the burden of disease remains high. An estimated 82.6 million American adults have vascular disease, with coronary heart disease (CHD) and stroke accounting for 16.3 and 7 million, respectively. Mortality data show that CVD accounted for $32.8 \%$ of all deaths in 2008 , or one of every three deaths in the USA. The total direct and indirect cost of CVD and stroke in the USA for 2008 is estimated to be USD $\$ 297.7$ billion. ${ }^{1}$ The AHA has put forth a goal to improve cardiovascular health in the USA by 2020 by reducing deaths from CVD and stroke by $20 \%$. By 2030 , the AHA estimates that $40.5 \%$ of the US population will have some form of CVD, with direct costs estimated at USD $\$ 818$ billion. ${ }^{2}$ Effective prevention strategies are needed if the growing burden of CVD is to be arrested.

\section{Brief pathophysiology of atherosclerosis}

Atherosclerosis is a multifactorial, multistep disease that involves chronic inflammation from initiation to progression. All the risk factors contribute to pathogenesis by aggravating the underlying inflammatory process. ${ }^{3}$ Destruction of endothelium leads to loss of antithrombic and fibrinolytic factors and nitrous oxide (NO), an increase in the production 
of vasoconstrictors (thromboxane A2 and prostaglandins), and an increase in intracellular calcium-derived vasoconstricting factors. ${ }^{3}$ Endothelial damage also causes platelets to aggregate at damaged sites, causing monocytes to enter the intima and proliferate. Intracellular lipid peroxidation leads to formation of lipoperoxides, which are toxic to plasma membranes and combine with apolipoprotein (apo) B and phospholipids to prevent low-density lipoprotein (LDL) from binding to the LDL receptor. LDL is oxidized and acts as an attractant to macrophages and monocytes. Macrophages ingest LDL and become foam cells, while monocyte mobility is hampered. Several other mechanisms have been implicated in the formation of atheromatous plaques, including imbalance between the coagulation cascade and fibrinolytic systems, accumulation of free radicals, certain infections and leukocytes and adhesion molecules. ${ }^{3}$ Elevated homocysteine in blood leads to thrombosis, intimal thickening, generation of free radicals, and alteration of the methylation status of genes.,

\section{Role of nitrous oxide}

Nitrous oxide and prostacyclin mediate vasodilatation of the endothelium. ${ }^{5}$ Nitric oxide (NO) is synthesized from the amino acid L-arginine in endothelial cells by nitric oxide synthase (NOS), which maintains vascular homeostasis by modulation of vascular dilator tone, regulation of local cell growth, and protection of the vessel from the injurious consequences of platelets and cells circulating in the blood. ${ }^{6}$ Conversion of angiotensin I to angiotensin II also acts as a vasoconstrictor, modulating the effect of the vasododilators. ${ }^{5}$

\section{Disruption of protective mechanisms}

Development of atherosclerosis leads to the disruption of the mentioned protective mechanisms inherent to the endothelium and causes the formation of plaque and thrombosis. Coronary artery occlusion is the direct effect of thrombus formation on ruptured and unruptured plaques at the site of atherosclerosis and is responsible for $60 \%-80 \%$ of acute coronary syndrome (ACS) cases. Inflammation-mediated neovascularization and intra-plaque hemorrhage, along with necrosis in the lipid core, are most often the cause of thrombogenesis. ${ }^{7}$ Rarely, genetic disorders like familial lecithin:cholesterol acyltransferase deficiency and fish-eye disease cause low-plasma high-density lipoprotein (HDL) and accumulation of unesterified cholesterol in the body. ${ }^{8}$

\section{Role of plaque formation}

Atherosclerosis with thrombus formation has been recognized as a major cause of cardiovascular death. It begins early in childhood and progresses in adult life when it can potentially manifest as coronary artery disease (CAD), stroke, and/ or peripheral vascular disease. Plaque formation is favored by a zone of absence of shear stress, which occurs mostly at arterial bifurcations. This is hypothesized to be related to the absence of elastin at this location and the presence of collagen-proteoglycan complexes, which causes LDL retention. ${ }^{9}$ Plaque rupture is the most common cause of arterial thrombosis coronary artery syndrome and is hypothesized to be a common cause of large-vessel atherosclerotic stroke. Anatomically, a ruptured plaque contains a large lipid-rich core and a thin fibrous cap with smooth muscles and macrophages, angiogenesis, adventitial inflammation, and remodeling. ${ }^{10}$

In a recent study of 697 patients, plaque morphology was studied in the setting of ACS. Stone et al evaluated these patients with three vessel angiograms and intravascular ultrasound (IVUS) and conducted follow-up for a median of 3.4 years. ${ }^{11}$ Adverse cardiac events occurred in distributions of previously "non-culprit" areas with fibroatheromas or a large plaque burden. ${ }^{11}$ Increases in the intimal and medial thickness of the carotid artery (measured by ultrasonography) are directly associated with an increased risk of myocardial infarction and stroke in adults $>65$ years old without a history of CVD. ${ }^{12}$

\section{Primary prevention}

Since atherosclerosis begins in childhood, primary prevention must begin as early as possible. To this end, screening, riskfactor reduction, and appropriate use of primary prevention medications are now being applied to children. The 2003 AHA guidelines for primary prevention of atherosclerosis beginning in childhood recommend that children older than 2 years with a family history of hyperlipidemia or premature cardiac disease should have targeted screening of fasting lipids. Concern should be raised if HDL levels are $<35 \mathrm{mg} / \mathrm{dL}$, total cholesterol is borderline $(>170 \mathrm{mg} / \mathrm{dL})$ or elevated $(>200 \mathrm{mg} / \mathrm{dL})$, low-density-lipoprotein cholesterol (LDL-C) is borderline $(>110 \mathrm{mg} / \mathrm{dL})$ or elevated $(>130 \mathrm{mg} / \mathrm{dL})$ or triglycerides are $>150 \mathrm{mg} / \mathrm{dL}$. Screening is also recommended if family history is unknown or other risk factors are present. ${ }^{13}$

The 2010 American College of Cardiology Foundation/ AHA guidelines recommend measurement of carotid artery intimal-medial thickness (CIMT) for cardiovascular risk assessment in asymptomatic adults at intermediate risk (Class IIa recommendation, level of evidence: B). ${ }^{14}$

\section{Role of screening tools}

Recently, in a National Institutes of Health-sponsored multiethnic study conducted to validate imaging for primary 
prevention of atherosclerosis, Zeb and Budoff showed that coronary artery calcium scores and CIMT evaluation are validated as a marker of atherosclerosis. ${ }^{16}$ Current national guidelines recommend using coronary artery calcium as a screening tool for risk stratification of intermediate-risk (10\%-20\% 10-year risk), asymptomatic population (Class IIa recommendation), and low- to intermediate-risk populations (6\%-10\% 10-year risk; level of evidence: B). ${ }^{14,16}$

The Screening for Heart Attack Prevention and Education (SHAPE) task force conducted noninvasive screening of asymptomatic men aged 45-75 years and asymptomatic women aged 55-75 years. The primary goal was to detect and treat all those with subclinical atherosclerosis except those considered very low risk. In addition to screening for risk-factor assessment (lifestyle, family history, blood pressure, diabetes, hyperlipidemia, etc), the authors advocate additional arterial structure testing, such as carotid ultrasound, coronary calcium score by computed tomography (CT) imaging, cardiac and aortic magnetic resonance imaging (MRI), and ankle-brachial index. ${ }^{17}$ Additional arterial function testing, such as ultrasound brachial vasoreactivity, as well as radial and fingertip tonometry, can also be considered.

In an observational study of 1118 Spanish patients over 30 years of age, Aguilar-Shea et al identified 320 subjects as low-intermediate cardiovascular risk using the European Systematic Coronary Risk Evaluation (SCORE) ${ }^{18}$ After B-mode ultrasound, $18.4 \%$ of the subjects were reclassified to the high-risk category, suggesting that CIMT measurement could be a useful preventive tool. ${ }^{18}$

An article by Hecht has further stressed the importance of imaging modalities in the primary prevention of atherosclerosis. ${ }^{19}$ Indeed, a focused analysis of vascular anatomy often leads to the recategorizing of the risk of asymptomatic individuals irrespective of their cholesterol levels.

\section{Role of estrogens}

Lenfant et al, in their review of the role of estrogens (especially 17-beta estradiol) in atherosclerosis in women have pointed out that the administration of exogenous estrogen closer to menopause may prevent CHD. ${ }^{20}$ They reviewed the vasculoprotective actions of estrogens, especially on stabilization of endothelium and prevention of early atheroma formation. However, the major side effects of estrogen-containing compounds, like hypercoagulability and endometrial and breast cancer, are still concerning. Currently, the use of estrogens is not recommended for cardioprotection. ${ }^{20}$

\section{Role of infection}

In a recent review by Deniset and Pierce, the role of Chlamydophila pneumoniae in the development of atherosclerosis was examined. Although there is experimental evidence to support the potential of $C$. pneumoniae to lead to development of atherosclerotic plaques, in none of the long-term studies in which antibiotics were used to treat a possible infectious cause of atherosclerosis was benefit of such treatment shown. ${ }^{21}$

\section{Role of antioxidants}

In recent years, there has been significant discussion about antioxidants and their possible role in prevention of atherosclerosis. The antioxidant market is a multi-billion-dollar industry, but there are no randomized clinical studies providing support that they are of benefit in the prevention of CVD. ${ }^{22}$

In the Coronary Artery Risk Development in Young Adults (CARDIA) study, the possible association between consumption of coffee, decaffeinated coffee, caffeine, and tea in young adulthood with the presence and progression of coronary artery-calcified plaque and CIMT later in life was evaluated. This cohort of 5115 adults aged 18-30 years old was followed at regular intervals for up to 20 years. It was concluded that, besides a nonsignificant inverse relationship between tea consumption and coronary artery calcification, no significant association could be found between caffeine intake and coronary or carotid atherosclerosis. ${ }^{23}$ The Biolmage study is an ongoing prospective observational study evaluating the associations among imaging and circulating biomarkers and their ability to predict atherothrombotic events in asymptomatic at-risk subjects for the primary prevention of myocardial infarction and stroke. ${ }^{24} \mathrm{~A}$ total of 7687 insured individuals were evaluated at baseline by randomization to telephone interview, baseline physical examination, or physical examination with evaluation of cardiovascular system by imaging modalities. Any abnormality in the latter group led to further investigation by contrast CT or MRI, or positron emission tomography (PET) scan. This ongoing study has as its end point 600 atherothrombotic events in the imaged group. DNA and RNA from blood has been stored for biomarker evaluation.

\section{Role of diet}

The American heart Association provides recommendations for diet and lifestyle modifications (Table 1). The importance of diet in cardiovascular health has been summarized by Dauchet et al. ${ }^{25}$ As noted in their review, most of the evidence supporting the cardio protective properties of fruits and vegetables is based on observational epidemiological studies, 
which have reported either weak or associations of low significance. Although fruit and vegetable consumption leads to lower blood pressure, little is known about its effect on lipids and blood sugar or whether it prevents plaque formation. Jabłecka et al treated 38 patients who had peripheral vascular disease with type II diabetes using L-arginine. ${ }^{26}$ The total study population was 50 (38 subjects and twelve controls). The results showed statistically significant increases in NO concentration and total antioxidant status levels in the treatment group, suggesting that L-arginine may have an antioxidative effect. The American Heart Association also provides guidelines for dietary modifications in the pediatric population (Table 2).

\section{Role of exercise}

It is now well accepted that a sedentary lifestyle increases the risk of atherosclerosis. The AHA guidelines recommend at least 150 minutes per week of moderate exercise or 75 minutes per week of vigorous exercise or a combination of moderate and vigorous activity. There are several mechanisms by which exercise promotes protection against atherosclerosis. Exercise reduces or prevents oxidative stress and inflammation through downregulation of endothelial angiotensin II type 1 receptor expression. This leads to decreases in nicotinamide adenine dinucleotide phosphateoxidase activity and superoxide anion production, which in turn decreases reactive oxygen species generation, preservation of endothelial NO bioavailability and its protective anti-atherogenic effects. This is caused by endothelial activation from laminar shear stress. Skeletal muscle contraction releases anti-inflammatory cytokines, such as interleukin-6, which inhibits tumor necrosis factor-alpha production in adipose tissue and macrophages. Exercise-associated weight loss also leads to a lowering of blood pressure, improvement in insulin sensitivity, and increased HDL. ${ }^{27} \mathrm{~A}$ recent review of several observational and interventional trials of the effects of physical activity on CVD has found that regular exercise leads to triglyceride reduction, apo $\mathrm{B}$ reduction, and HDL increase. In addition, physical activity can also lead to an increase in tissue plasminogen activator activity and a decrease in coronary artery calcium. ${ }^{28}$

As mentioned, risk-factor assessment is the first step in the primary prevention of atherosclerotic disease. The next stage of evaluation could include blood tests like lipid profile as well as new markers like C-reactive protein (CRP), lipoprotein-associated phospholipase A2, LDL subfraction analysis, apo B/apo A-I ratio, and lipoprotein A. Assessment of coronary calcification and CIMT contribute to risk stratification. There is also suggestion that influenza vaccine may have a preventive effect and that gene therapy with genetic subtyping and proteomics may lead to new preventive clinical insights. ${ }^{29}$

\section{Secondary prevention Recommendations of the AHA and American Stroke Association}

The AHA and American College of Cardiology Foundation have provided recommendations for secondary prevention and risk reduction in patients with atherosclerotic disease. These include smoking cessation, a blood pressure goal of 140/90 mmHg, LDL-C $<100 \mathrm{mg} / \mathrm{dL}(<70 \mathrm{mg} / \mathrm{dL}$ in high-risk patients), and body mass index 18.5 to $24.9 \mathrm{~kg} / \mathrm{m}^{2}$. The AHA also recommends daily aspirin (or clopidogrel), renin-angiotensin-aldosterone blockers and beta-blockers for secondary prophylaxis. Screening for depression, especially in post-cardiac bypass patients and cardiac rehabilitation has also been strongly recommended. ${ }^{31}$ Depression has high prevalence among patients with CHD and is independently associated with increased cardiovascular mortality and morbidity. The AHA recommends screening for depressive symptoms in patients with CHD. ${ }^{32}$ Further, the AHA strongly recommends exercise-based cardiac rehabilitation, deeming it relatively safe as long as it is medically supervised and physician directed. Cardiac rehabilitation significantly reduces total and cardiac mortality, when compared with patients who do not undergo such programs. Several mechanisms have been implicated in the protective mechanism of exercise. These include improved endothelial function; enhanced synthesis, release, and duration of action of NO; and anti-inflammatory effects and/or lowering of CRP levels. Exercise also leads to reduction in weight, blood pressure, and triglycerides and elevation in HDL. The AHA also mentions that endurance training has anti-ischemic, antiarrhythmic, and anti-thrombotic properties. ${ }^{33}$

The 2011 American Stroke Association guidelines on the management of patients with extracranial carotid and vertebral artery disease strongly recommend risk-factor reduction. ${ }^{34}$ This includes cessation of cigarette smoking and treatment of hypercholesterolemia, diabetes, and hypertension. In this guideline, antiplatelet therapy with aspirin, aspirin plus dipyridamole, or clopidogrel (when aspirin cannot be used), is recommended. Carotid endarterectomy or stenting is recommended for symptomatic patients with 50\%-99\% stenosis and asymptomatic patients with 70\%-99\% stenosis. Revascularization of the brachiocephalic and subclavian artery 
using balloon angioplasty, bypass grafting, atherectomy, and stenting should be considered in symptomatic patients. ${ }^{34}$ Table 3 outlines the recommended goals for lipid management.

\section{Role of newer antiplatelet agents}

Ticagrelor is a reversible direct inhibitor of adenosine diphosphate receptor P2Y12 that has a more rapid onset and more pronounced platelet inhibition than clopidogrel. The PLATO study compared ticagrelor (180 mg loading dose, $90 \mathrm{mg}$ twice daily thereafter) and clopidogrel (300-600 mg loading dose, $75 \mathrm{mg}$ daily thereafter) in a multicenter double-blind randomized trial for the prevention of cardiovascular events in 18,624 patients with an ACS, with or without ST-segment elevation. ${ }^{35}$ The study found that, in comparison with clopidogrel, ticagrelor significantly reduced the rate of death from vascular causes, myocardial infarction, or stroke, without an increase in the rate of overall major bleeding. However, more instances of fatal intracranial bleeding were observed with patients taking ticagrelor. ${ }^{35}$ The TRITON-TIMI 38 study compared prasugrel (60 mg loading dose then $10 \mathrm{mg}$ daily maintenance dose) with clopidogrel (300 mg loading dose then $75 \mathrm{mg}$ daily maintenance dose) in a randomized study of 13,608 patients for 6 to 15 months. The investigators found that in patients with an ACS with scheduled percutaneous coronary intervention (PCI), prasugrel therapy was associated with significantly reduced rates of ischemic events, including stent thrombosis, but with an increased risk of major bleeding, including fatal bleeding. There was no difference in overall mortality between the two treatment groups. ${ }^{36}$

\section{Secondary prevention in diabetic patients}

In diabetic patients with $\mathrm{CAD}$, progression of atherosclerosis was studied in the APPROACH trial. ${ }^{37}$ In this study, rosiglitazone's effect on coronary atherosclerosis, as assessed by IVUS, was compared with that of glipizide. APPROACH was a randomized double-blind controlled 18-month study of 672 patients aged 30 to 80 years with established type 2 diabetes mellitus. Patients were treated with lifestyle changes, one oral agent, or submaximal doses of two oral agents. Patients had at least one atherosclerotic plaque with $10 \%-50 \%$ luminal narrowing in a coronary artery and had not undergone intervention during a coronary angiography. The primary outcome was change in atheroma volume in naïve arteries. A secondary outcome was change in total atheroma volume (TAV) in the baseline segment with greatest disease. Compared with glipizide, rosiglitazone did not significantly reduce atherosclerosis volume as $(-0.64 \%$; $95 \%$ confidence interval $[\mathrm{CI}],-1.46$ to $0.17 ; P=0.12$ ). However, secondary outcomes of normalized TAV was significantly reduced by rosiglitazone compared with glipizide $\left(-5.1 \mathrm{~mm}^{3} ; 95 \%\right.$ CI, -10.0 to $-0.3 ; P=0.04)$; but no significant difference between groups was observed for the change in TAV within the most diseased baseline $10 \mathrm{~mm}$ segment $\left(-1.7 \mathrm{~mm}^{3} ; 95 \%\right.$ CI, -3.9 to $0.5 ; P=0.13) .{ }^{37}$

In a sub-analysis of the APPROACH study, ${ }^{38}$ the medium-term effect of rosiglitazone and glipizide on intrastent neointima hyperplasia was studied. Restenosis pattern, as determined by IVUS and quantitative coronary angiography, was assessed in these patients with type II diabetes mellitus and CAD. A total of 462 patients were randomized to rosiglitazone or glipizide for up to 18 months, with baseline and follow-up IVUS examinations. Although this study did not show any difference between the size of plaque after stenting, it did demonstrate reduction in plaques at 18 months in the group with bare metal stents $\left(-5.6 \mathrm{~mm}^{3} \mathrm{vs} 1.9 \mathrm{~mm}^{3}\right.$; $P=0.61)$. Similarly, there was no significant difference in percentage intimal hyperplasia volume between the rosiglitazone and glipizide groups at 18 months among those treated with a bare metal stent $(24.1 \%$ vs $19.8 \% ; P=0.38)$ or with a drug-eluting stent $(9.8 \%$ vs $8.3 \% ; P=0.57)$. There was no difference in angiography data between the two treatment groups in this study. Thus, both drugs appeared to offer benefit in neointimal growth. ${ }^{38}$

\section{Plaque regression}

It is now known that plaques are able to regress. Plaque reversal occurs by removal of lipids and necrotic material, endothelial repair, or halt of vascular smooth muscle cell proliferation. Several mechanisms explain this reversal, such as high-density lipoprotein cholesterol (HDL-C) action, destruction of foam cells and macrophages in lymph nodes and restoration of endothelium by neighboring cells or circulating progenitors. ${ }^{39}$

\section{Role of statins, phosphodiesterase inhibitors, and thiazolidinediones}

In a recent meta-analysis of eight trials, 919 patients (461 patients in the statin group and 458 in the placebo group) were studied with IVUS. ${ }^{40}$ There was no significant difference between the two groups in terms of their plaque characteristics at baseline. However, there was a statistically significant mean difference in coronary atheroma volume between the statin therapy and the placebo arms, which was -3.573 (95\% CI -4.46 to $-2.68 ; P<0.01)$. This suggests that statins have the potential to induce plaque reversal. 
One small study analyzed the effects of rosuvastatin combined with ramipril on atheroma volume and its mechanism in patients with intermediate CAD ${ }^{41}$ In this study, 21 patients received rosuvastatin (20 $\mathrm{mg}$ daily) and 19 patients received rosuvastatin along with ramipril $(20 \mathrm{mg}$ and $10 \mathrm{mg}$, respectively) for 9 to 12 months. The study focused on measurement of TAV per $10 \mathrm{~mm}$ segment along with lipids, metabolic parameters (adiponectin, insulin sensitivity), and biomarkers (high-sensitivity [hs]-CRP, matrix metalloproteinase-9) at baseline and end of study. There was decrease in the TAV in both groups, with reduction in the CRP levels in the combination treatment group.

Takayama and colleagues studied the effect of rosuvastatin on plaque volume on 126 patients with stable CAD on lipid-lowering therapy at 37 centers in a 76-week open-label study. ${ }^{42}$ Subjects received rosuvastatin $2.5 \mathrm{mg}$ per day (with the potential to be increased at 4-week intervals to up to $20 \mathrm{mg}$ per day). The primary end point of percent change of plaque volume, as evaluated by IVUS was $-5.1 \% \pm 14.1 \%$ in the rosuvastatin group $(P<0.0001)$.

Hibi et al studied effects of statin treatment on plaque regression in patients with polyvascular disease versus those with CAD alone. ${ }^{43}$ They studied 252 patients (at 33 centers) with a history of an ACS, who underwent percutaneous intervention to localize the lesion followed by treatment with atorvastatin (20 mg per day) or pitavastatin (4 mg per day). Both groups showed regression of plaques, as assessed by IVUS at baseline and at 8-12 months follow-up. A subanalysis of this study showed that aggressive lipid lowering by pitavastatin and atorvastatin results in marked regression of atherosclerotic coronary lesions after ACS. The study involved data from 251 patients ( 73 were diabetic) and the authors studied the association of lipid levels after statin therapy with regression of atherosclerotic coronary lesions and major cardiovascular events in patients after ACS. The results showed that decrease in LDL-C, non-HDL-C, LDL-C/ HDL-C ratio, and apo B levels were all associated with a progressively smaller plaque burden. In diabetic patients, further reduction of these parameters was associated with a significantly greater reduction in plaque volume. ${ }^{44}$

In a prospective randomized comparative study using rosuvastatin $20 \mathrm{mg}(\mathrm{n}=65)$ and atorvastatin $40 \mathrm{mg}(\mathrm{n}=63)$, IVUS was used at baseline and at 11-month follow-up, to show effective plaque regression. TAV and percent atheroma volume (PAV) was measured. Plaque was decreased in 99 of 128 patients (77\%; 85\% [55/65] in the rosuvastatin group vs $70 \%[44 / 63]$ in the atorvastatin group). Both groups showed change in TAV: $-4.4 \pm 7.3 \mathrm{~mm}^{3}$ for the rosuvastatin group and $-3.68 \pm 6.8 \mathrm{~mm}^{3}$ for the atorvastatin group $(P=0.5)$. The difference in PAV between the two groups was not statistically significant $(P=0.14)$. These results demonstrate that both statins are effective in reducing plaque burden. ${ }^{45}$

Tani et al studied the effect of pravastatin on atherosclerotic plaque in a 6-month prospective study of 64 patients. Pravastatin reduced plaque volume, as measured by volumetric intravascular ultrasonography, by $12.6 \%$ $(P<0.0001$ vs baseline $) .{ }^{46}$ The authors also found a significant reduction in the apoB/apoA-1 ratio $(P<0.0001$ vs baseline).

The effect of atorvastatin on MRI changes in atherosclerotic plaques in the thoracic and abdominal aorta was studied in a prospective study in which 87 patients with hypercholesterolemia were administered either atorvastatin $(n=42)$ or atorvastatin plus etidronate $(n=47)$ for 12 months. ${ }^{47} \mathrm{LDL}$ levels and wall thickness in the thoracic aorta were reduced in both groups $(-15 \%$ and $-14 \%$ for the combination therapy and monotherapy group, respectively; $P<0.001$ vs baseline for both groups). However, only patients in the combination group had a reduction in wall thickness of the abdominal aorta $(-14 \% ; P<0.001$ vs baseline).

The SECURE study was an ongoing Phase IV doubleblind randomized controlled multicenter clinical trial of patients who were given either a combination of cilostazol and probucol or cilostazol alone. ${ }^{48}$ Plaque volume and composition using IVUS was studied as the primary end point at baseline and 9-month follow-up. This study has been completed and results are awaited.

Kovarnik et al randomized 89 patients to receive either atorvastatin $80 \mathrm{mg}$ plus ezetimibe $10 \mathrm{mg}$ or standard treatment per the patients' general practitioner for 12 months. ${ }^{49}$ The authors found a decrease in the coronary artery PAV $(-0.4 \%)$ in the group on combination treatment versus an increase $(+1.4 \%)$ in the other group $(P=0.014)$, as measured by IVUS. There was also an increased frequency of combined atherosclerosis regression (increased lumen volume plus decreased PAV) in patients taking both medications (40.5\%) compared with the group on monotherapy (14.9\%) $(P=0.007)$.

A recent study used cardiovascular magnetic resonance (MR) to study volumetric carotid plaque measurement on 26 subjects who had carotid plaques greater than $1.1 \mathrm{~mm}$ and coronary or cerebrovascular atherosclerotic disease (mean age $67 \pm 2$ years, 7 females). ${ }^{50}$ The subjects underwent evaluation with 3 Tesla MR (T1, T2, proton density, and time of flight sequences) and two-dimensional ultrasound at baseline and after 6 months of statin therapy. Plaque volume 
as measured by MR decreased by $5.8 \% \pm 2 \%$ (1036 \pm 59 to $\left.976 \pm 65 \mathrm{~mm}^{3} ; P=0.018\right)$ while mean plaque volume, as measured by ultrasound, was unchanged $(1.12 \pm 0.06$ vs $1.14 \pm 0.06 \mathrm{~mm} ; P=$ not significant $)$. Those patients $(\mathrm{n}=13)$ who had an initiation or increase of statins had $-8.8 \% \pm 2.8 \%$ change $(P=0.001)$, as compared with an insignificant change in patients $(\mathrm{n}=13)$ who were on statin maintenance.

Nicholls et al compared serial intravascular ultrasonography in 1039 patients with coronary disease, at baseline and after 104 weeks of treatment with either atorvastatin, $80 \mathrm{mg}$ daily, or rosuvastatin $40 \mathrm{mg}$ daily. ${ }^{51} \mathrm{In}$ the atorvastatin group, the atheroma volume decreased by $0.99 \%$ (95\% CI -1.19 to $-0.63)$ and $1.22 \%(95 \% \mathrm{CI}-1.52$ to -0.90$)$ in rosuvastatin group $(P=0.17)$. The normalized TAV was $-6.39 \mathrm{~mm}^{3}(95 \%$ $\mathrm{CI},-7.52$ to -5.12$)$ with rosuvastatin and $-4.42 \mathrm{~mm}^{3}(95 \%$ CI, -5.98 to -3.26$)$ with atorvastatin $(P=0.01)$. The sideeffect profiles were acceptable in both groups.

A previous review of four prospective randomized trials showed significant regression in coronary atherosclerosis associated with the lowering of LDL-C levels and an increase in HDL. ${ }^{52}$ This post-hoc analysis included 1455 patients with angiographic CAD who underwent serial intravascular ultrasonography while receiving statin treatment for 18 to 24 months.

In their 6-month study, Yang and colleagues studied the effect of pioglitazone on coronary plaque area and plaque burden in patients with impaired glucose tolerance and $\mathrm{CAD}$ who were taking atorvastatin $20 \mathrm{mg}$ daily for 3 months, followed by $10 \mathrm{mg}$ daily for the next 3 months. ${ }^{53}$ At the completion of treatment, they underwent IVUS. Compared with the control group, 6 months' treatment with pioglitazone significantly decreased coronary plaque burden $(50.7 \pm 11.1$ vs $64.1 \% \pm 10.3 \% ; P<0.05)$, decreased plaque area $(6.22 \pm 2.03$ vs $8.31 \pm 4.29 ; P<0.05)$, decreased thincap fibroatheroma prevalence ( $11 \%$ vs $22 \% ; P<0.05$ ), and decreased percentage of necrotic core area $(16 \% \pm 8 \%$ vs $31 \% \pm 7 \% ; P<0.05)$. Incidentally, the patients taking pioglitazone had significantly lower hs-CRP and endothelin-1 levels and higher adiponectin levels.

Another study of 26 patients evaluated the effect of pioglitazone on coronary plaque structure. IVUS was used to demonstrate that pioglitazone reduced plaque burden without LDL-C reduction in patients suffering from diabetes and impaired glucose tolerance. ${ }^{54}$ Thirteen patients received pioglitazone $15 \mathrm{mg}$ per day for an initial 14 days after percutaneous coronary intervention followed by $30 \mathrm{mg}$ per day with the remaining patients as control. At the end of 6 months, the pioglitazone group had significantly reduced plaque volume $\left(101.3 \pm 32.1\right.$ to $\left.94.6 \pm 33.6 \mathrm{~mm}^{3},-7.2 \% ; P=0.0023\right)$. Serum cholesterol levels were also significantly improved in the pioglitazone group; they had lower triglyceride and CRP levels and higher HDL levels at the end of the study.

Yet another study has shown significant reduction in plaque volume with the use of pioglitazone and statin combination therapy using IVUS and IVUS-virtual histology. ${ }^{55}$ Analysis of 29 plaques in 25 diabetic patients who were treated with $80 \mathrm{mg}$ of atorvastatin plus $30 \mathrm{mg}$ of pioglitazone daily for 6 months was performed. Mean elastic external membrane volume was significantly reduced between baseline and follow-up (343.9 vs $\left.320.5 \mathrm{~mm}^{3} ; P<0.05\right)$, as was mean TAV (179.3 vs $\left.166.6 \mathrm{~mm}^{3} ; P<0.05\right)$. Change in TAV showed a $6.3 \%$ mean reduction. However, areas of the necrotic core increased from $9 \%$ to $14 \%(P<0.05)$ in spite of fibrous tissue and calcium decreasing over the 6 months of follow-up.

In a prospective study of 140 patients aged 50-75 years old (64 males and 76 females), the effects of aggressive lipid lowering with atorvastatin on the echogenicity of carotid plaques was studied. All subjects had moderate-grade carotid artery disease (symptomatic and asymptomatic) but without the indication for surgical intervention and did not suffer from CHD, renal failure, hypothyroidism, or osteoporosis. Group A $(n=70)$ received atorvastatin $10 \mathrm{mg}$ or $20 \mathrm{mg}$ (target LDL-C $<100 \mathrm{mg} / \mathrm{dL})$ and Group $\mathrm{B}(\mathrm{n}=70)$ received atorvastatin $80 \mathrm{mg}$ (target LDL-C $<70 \mathrm{mg} / \mathrm{dL}$ ). There were no significant differences between groups at baseline and none of the subjects had ongoing use of statins. Group B had a higher augmentation of the grayscale median on carotid ultrasound at the end of 12 months (from 66.39 \pm 23.66 to $100.4 \pm 25.31$ ) than group A (from $64.4 \pm 23.62$ to $85.39 \pm 20.21)(P=0.024)$. No change in the degree of carotid stenosis was noted in either treatment arm. Along with enhanced carotid plaque echogenicity, atorvastatin showed significant reduction in serum hs-CRP, osteopontin, and osteoprotegrin (novel cardiovascular biomarkers belonging to the tumor necrosis factor family) levels in a dose-dependent manner $(P<0.001) .{ }^{56}$ In the 2 -year Dietary Intervention Randomized Controlled Trial - Carotid (DIRECT-Carotid) study, participants were randomized to a low-fat, Mediterranean, or low-carbohydrate diet. The change in carotid plaque caliber was evaluated using CIMT (measured with standard B-mode ultrasound) and carotid vessel wall volume (VWV; measured with carotid three-dimensional ultrasound). After 2 years of dietary intervention, the observation was a significant $5 \%$ regression in mean carotid VWV $\left(-58.1 \mathrm{~mm}^{3} ; 95 \%\right.$ CI -81.0 to $\left.-35.1 \mathrm{~mm}^{3} ; P<0.001\right)$, indicating decreased 
atherosclerosis. There was no differences among the lowfat, Mediterranean, or low-carbohydrate groups (-60.69, -37.69 , and $-84.33 \mathrm{~mm}^{3}$, respectively; $P=0.28$ ) and mean change in intima-media thickness was $-1.1 \%(P=0.18)$. Those participants who achieved greater weight loss exhibited greater decrease in carotid VWV regression (mean decrease, $-128.0 \mathrm{~mm}^{3} ; 95 \% \mathrm{CI}-148.1$ to $-107.9 \mathrm{~mm}^{3}$ ) than those who exhibited progression (mean increase, $+89.6 \mathrm{~mm}^{3}$; $95 \% \mathrm{CI}+66.6$ to $\left.+112.6 \mathrm{~mm}^{3}\right) .{ }^{57}$

Nanomedical advances may have a role as approaches to reversing plaque formation. ${ }^{58}$ Nanotechnology has been primarily investigated as an enhancement of stent technology. Lobatto et al reviewed the anti-atherotic properties of liposomes, which are artificially prepared spherical self-closed structures formed by lipid bilayers that have an aqueous interior. ${ }^{59}$ Since their discovery in the 1960 s, they have been extensively researched as drug carriers. Liposomes are currently being investigated for several therapeutic strategies such as plaque reversal and lipid-lowering, antiinflammatory, and anti-restenotic therapies. ${ }^{59}$ Nanoparticles have been studied as potential anti-restenotic drug-delivery systems after angioplasty, with the target molecules being the extracellular matrix and collagen in the vascular basement membrane..$^{60,61}$

\section{Role of HDL}

It is now well established that HDL has athero-protective properties. Epidemiological studies have shown that decreased levels of HDL-C is an independent inverse predictor of CAD, even in patients with normal levels of LDL-C. ${ }^{62,63}$ According to one study, an estimated $40 \%$ of patients with premature CAD have low levels of HDL-C and the risk of CAD increases by $1 \%-3 \%$ for every $1 \%$ reduction in HDL-C levels. ${ }^{64}$

\section{Cholesteryl ester transfer protein (CETP) inhibitors}

A new class of drugs, the cholesteryl ester transfer protein inhibitors (CETP-Is), may have plaque reversal properties. CETP's constitutive role is to transfer cholesterol esters from HDL-C to LDL-C and very low-density lipoprotein. This leads to lower concentrations of HDL-C but higher concentrations of LDL-C and very low-density lipoprotein cholesterol, which are pro-atherogenic. Hence, CETP-Is have a potential therapeutic effect in atherosclerosis treatment. Studies are underway to test this hypothesis. The dal-PLAQUE study demonstrated plaque reversal by dalcetrapib, a cholesterol ester transfer protein modulator. ${ }^{65}$ This 2-year multicenter Phase IIB double-blind randomized placebo-controlled study of 130 patients compared the efficacy of dalcetrapib (600 mg/day) with placebo. The effect on atherosclerosis was evaluated using MRI and fluorine-18 fluorodeoxyglucose-PET/CT at 12 and 6 months, respectively. The results showed that the MRI-derived change in total vessel area was reduced in patients treated with dalcetrapib compared with those given placebo. The absolute change from baseline relative to placebo was $-4.01 \mathrm{~mm}^{2}$ (90\% 95\% CI -7.23 to -0.80 ; nominal $P=0.04)$. The carotid artery analysis showed a $7 \%$ reduction in the dalcetrapib group compared with the placebo group $(-7.3$ [90\% CI -13.5 to -0.8$]$; nominal $P=0.07$ ). The frequency of adverse events was similar in both groups.

Recently, a Phase I study of 38 subjects showed that the CETP-I BAY 60-5521 is clinically safe and well tolerated. No effects on heart rate, blood pressure, or electrocardiography recordings were observed and a clear pharmacodynamic effect on CETP inhibition and HDL-C was demonstrated. Unfortunately, plaque burden was not assessed. ${ }^{66}$

The safety and efficacy profile of anacetrapib, a highly potent CETP-I, has been characterized in recent studies. It does not appear at the moment that anacetrapib exhibits the side effects typical of an earlier CETP-I, torcetrapib. ${ }^{67}$ The results from the DEFINE study will determine the efficacy and tolerability of anacetrapib. ${ }^{68}$

\section{Role of niacin}

In a multi-arm study, Chen et al concluded that a combination of extended-release (ER) niacin and laropiprant (LRP), a $\mathrm{DP}_{1}$ antagonist that reduces vasodilation, was superior to atorvastatin in raising HDL levels after 12 weeks of treatment in 2340 patients with mixed hyperlipidemia. ${ }^{69}$ Previously, the ARBITER study had shown a slowing down of atherosclerosis in patients treated with ER niacin in addition to statin treatment, based on CIMT. ${ }^{70}$ When added to existing statin treatment, niacin was shown to be superior to ezetimibe in reducing CIMT in 315 patients. $^{71}$ Yadav et al have recently evaluated the safety and tolerability reports of ER niacin with LRP in existing literature and found the combination safe as add-on therapy to statins, allowing higher doses of niacin to be administered. ${ }^{72}$ The HPS2-THRIVE, a Phase III study, will assess the effects of raising HDL-C with ER niacin/LRP 2 gm versus matching placebo on the risk of heart attack or coronary death, stroke, or the need for arterial bypass procedures in people with a 
history of circulatory problems. ${ }^{73}$ The AIM-HIGH carotid MRI study is currently investigating if treatment with simvastatin plus niacin compared with simvastatin alone more effectively reduces the plaque fat content assessed by MRI and, therefore, further reduces heart attacks and strokes. ${ }^{74}$ This study is also investigating the effect of simvastatin plus niacin on plaque inflammation and HDL oxidation and proteomics. $^{75}$

\section{Role of peroxisome proliferator-activated receptor alpha/gamma agonists}

Buse et al has shown a beneficial effect of muraglitazar on raising HDL levels. ${ }^{76}$ Similarly, tesaglitazar at doses $\geq$ $1.0 \mathrm{mg}$ daily, significantly increased HDL-C versus pioglitazone $45 \mathrm{mg}$ daily $(P<0.001)$ in a 12 -week multicenter international randomized parallel-group trial including 500 type 2 diabetics aged 30-80 years old. ${ }^{77}$ Ragaglitazar also showed improvement in HDL levels of up to $31 \%$ in 177 hypertriglyceridemic type 2 diabetic subjects who participated in a 12-week double-blind parallel randomized placebo-controlled dose-ranging study. ${ }^{78}$

\section{Role of parenteral HDL therapy}

Reconstituted HDL infusions have recently shown significant improvement in plaque characterization index and coronary score on quantitative coronary angiography. The investigators of the ERASE study studied the effects of reconstituted HDL on plaque burden, as assessed by IVUS. ${ }^{79}$ This randomized placebo-controlled trial included 60 patients who were randomly assigned to receive one transfusion every week for 4 weeks of placebo (saline), 111 to receive $40 \mathrm{mg} / \mathrm{kg}$ of reconstituted HDL (CSL-111), and twelve to receive $80 \mathrm{mg} /$ $\mathrm{kg}$ of CSL-111. The percentage change in atheroma volume was $-3.4 \%$ with CSL-111 and $-1.6 \%$ for placebo $(P=0.48$ between groups, $P<0.001$ vs baseline for CSL-111). The nominal change in plaque volume was $-5.3 \mathrm{~mm}^{3}$ with CSL111 and $-2.3 \mathrm{~mm}^{3}$ with placebo $(P=0.39$ between groups, $P<0.001$ vs baseline for CSL-111). Waksman et al studied the effects of one treatment every week for a total of seven treatments over 7 weeks of autologous infusions of selective HDL delipidated plasma in patients with ACS. Plasma-selective delipidation converts alpha-HDL to pre-beta-like HDL, the most effective form of HDL for lipid removal from arterial plaques. Although the atheroma volume was not significantly different from that of the placebo group $(P<0.268)$, HDL infusion did reduce TAV, whereas this increased in the placebo group ${ }^{80}$ The CHI SQUARE Trial is an ongoing study to assess
Table I Diet and lifestyle recommendations for cardiovascular disease risk reduction

- Achieve and maintain a healthy body weight

- Balance calorie intake and physical activity

- Diet should be rich in vegetables and fruits

- Choose whole-grain, high-fiber foods

- Fish (especially oily fish) should be consumed at least twice a week

- Saturated fat intake should be $<7 \%$, trans fat to $<1 \%$, and cholesterol to $<300 \mathrm{mg} / \mathrm{d}$

- Lean meats and vegetables should be preferred

- Fat-free (skim), I\% fat, and low-fat dairy products should be preferred

- Intake of partially hydrogenated fats should be minimized

- Intake of products with added sugars should be minimized

- Little or no salt should be used to cook food

- Alcohol should be consumed in moderation

- Preferably, the AHA diet and lifestyle recommendations should be adhered to when away from home

Modified from "AHA 2006 diet and lifestyle recommendations for cardiovascular disease risk reduction" in Gidding SS, Lichtenstein AH, Faith MS, et al. Implementing American Heart Association pediatric and adult nutrition guidelines: a scientific statement from the American Heart Association Nutrition Committee of the Council on Nutrition, Physical Activity and Metabolism, Council on Cardiovascular Disease in the Young, Council on Arteriosclerosis, Thrombosis and Vascular Biology, Council on Cardiovascular Nursing, Council on Epidemiology and Prevention, and Council for High Blood Pressure Research. Circulation. 2009; 1 19(8):1 16I-1 175. ${ }^{15}$

Abbreviation: AHA, American Heart Association.

the effects of weekly injections of CER-001, an apo A-I-based HDL mimetic, on indices of atherosclerotic plaque progression and regression as assessed by IVUS measurements in patients with ACS. This Phase II multicenter double-blind ascendingdose placebo-controlled dose-finding trial includes subjects

Table 2 Pediatric dietary changes for individuals $>2$ years of age

- Dietary calories should be balanced with physical activity to maintain normal growth

- At least $60 \mathrm{~min} /$ day of moderate to vigorous play/physical activity should be encouraged

- Daily vegetables and fruits and limited juice intake

- Use vegetable oils and soft margarines low in saturated fat and trans fatty acids instead of butter or most other animal fats in the diet

- Eat whole-grain breads and cereals

- Avoid sugar-sweetened beverages and foods

- Consume nonfat (skim) or low-fat milk and dairy products daily

- Encourage oily fish intake, broiled or baked

- Reduce daily salt intake

Modified from "AHA pediatric dietary strategies for individuals $>2$ years of age: recommendations to all patients and families" in Gidding SS, Lichtenstein AH, Faith MS, et al. Implementing American Heart Association pediatric and adult nutrition guidelines: a scientific statement from the American Heart Association Nutrition Committee of the Council on Nutrition, Physical Activity and Metabolism, Council on Cardiovascular Disease in the Young, Council on Arteriosclerosis, Thrombosis and Vascular Biology, Council on Cardiovascular Nursing, Council on Epidemiology and Prevention, and Council for High Blood Pressure Research. Circulation. 2009; | 19(8): I |61-1 $175 .{ }^{.5}$

Abbreviation: AHA, American Heart Association. 
Table 3 Goals for lipid management

\section{For all individuals}

- Dietary therapy: reduce intake of saturated fats (to $<7 \%$ of total calories), trans fatty acids, and cholesterol (to $<200 \mathrm{mg} / \mathrm{d}$ ). I (B)

- Daily physical activity goal: At least 30 minutes, 7 days per week (minimum 5 days per week). Weight management goal: Body mass index $18.5 \mathrm{~kg} / \mathrm{m} 2$ to $24.9 \mathrm{~kg} / \mathrm{m} 2$. Waist circumference: women $<35$ inches $(<89 \mathrm{~cm})$, men $<40$ inches $(<102 \mathrm{~cm})$. I (B)

- Increased consumption of omega-3 fatty acids in the form of fish or in capsule form ( $\mathrm{l} \mathrm{g} / \mathrm{d}$ ) for risk reduction. Higher doses may be necessary for treatment of elevated triglycerides. Ilb (B)

\section{For lipid management}

Fasting lipid profile should be checked in all patients, and within 24 hours of hospitalization for those with an acute cardiovascular or coronary event

Lipid-lowering therapy should be initiated prior to discharge from hospital based on lipid profile

- LDL-C should be $<100 \mathrm{mg} / \mathrm{dL}$ (I [A]) and reduction to $<70 \mathrm{mg} / \mathrm{dL}$ is reasonabe in very high risk patients. Ila $(C)$

- If on-treatment LDL-C is $\geq 100 \mathrm{mg} / \mathrm{dL}$, intensify therapy (may require combination therapy). Ila (B)

- If triglycerides are 200 to $499 \mathrm{mg} / \mathrm{dL}$, non-HDL-C should be $<130 \mathrm{mg} / \mathrm{dL}$. I (B), if $>500 \mathrm{mg} / \mathrm{dL}$, fibrate therapy should be added to statin therapy to prevent acute pancreatitis I (C)

- If standard therapy fails, options to reduce non-HDL-C are

- Higher dose LDL-C-lowering therapy I (B), or

- Niacin Ila (B), or

- Fibrate therapy lla (B)

Note: Level of evidence is shown in brackets.

Modified from Smith SC Jr, Benjamin EJ, Bonow RO, et al. AHA/ACCF Secondary Prevention and Risk Reduction Therapy for Patients with Coronary and other Atherosclerotic Vascular Disease: 201 I update: a guideline from the American Heart Association and American College of Cardiology Foundation. Circulation. 20II Nov 29; I 24(22):2458-2473.

Abbreviations: HDL-C, high-density lipoprotein cholesterol; LDL-C, low-density lipoprotein cholesterol.

with ACS and has as its primary aim the measuring of absolute change in total plaque volume (assessed by intravenous ultrasound), from baseline to follow-up at about 3 weeks following the final dose of study medication (approximately 9 weeks after the baseline assessment). The secondary goal is percent change in total plaque volume. ${ }^{81}$

In a Phase IIB multicenter double-blind randomized parallel-group placebo-controlled study, the early effects of apo A-I synthesis with RVX000222 (100 mg twice daily) on low HDL-C levels are being studied. ${ }^{82}$ Using IVUS, the total and percent atheroma values at 26 weeks will be calculated. This study is based on the recent advances with synthetic HDL particles and recombinant apo A-I that have shown that HDL has the capacity to reverse coronary atherosclerosis. RVX000222 is a novel small molecule that increases plasma levels of HDL through increased apo A-I transcription.

\section{Conclusion}

The formation and progression of plaque is a complex process that involves inflammation at every step of the cascade. There is continued interest in the mechanisms involved in plaque pathophysiology and a great deal of research effort is being targeted at understanding the complexities of this disease. CVD has consistently remained a huge social burden and several national organizations have laid out clear and concise recommendations for the prevention and treatment of diseases related to it.

Further, the prevention of atherosclerosis has been an area of major interest that has led to increased awareness to start early in childhood. Over the last few decades, newer therapeutic interventions have been identified for the prevention and treatment of atherosclerotic disease. Most of them target one or more steps involved in the pathogenesis of atherosclerosis. These modalities range from changes in diet to cutting-edge use of nanotechnology. Statins appear to play a significant beneficial role in this arena and most of the recent research has been focused on the role of this group of drugs in the reversal of plaque. As newer medications are introduced to the list of treatment options, the future use of these interventions would have to be patient specific. It is likely that optimal therapy to achieve desired outcome will be a combination approach that includes pharmacologic, non-pharmacologic, and surgical options.

\section{Disclosure}

The authors declare no conflicts of interest in this work. The opinions expressed herein by the authors belong only to them. They should not be interpreted as being those of, or endorsed by, the Uniformed Services University of the Health Sciences, the US Army, the US Department of Defense, or the federal government.

\section{References}

1. Roger VL, Go AL, Lloyd-Jones DM, et al. American Heart Association Statistics Committee and Stroke Statistics Subcommittee. Executive summary: heart disease and stroke statistics - 2012 update: a report from the American Heart Association. Circulation. 2012;125:188-197.

2. Heidenreich PA, Trogdon JG, Khavjou OA, et al. American Heart Association Advocacy Coordinating Committee; Stroke Council; Council on Cardiovascular Radiology and Intervention; Council on Clinical Cardiology; Council on Epidemiology and Prevention; Council on Arteriosclerosis; Thrombosis and Vascular Biology; Council on Cardiopulmonary; Critical Care; Perioperative and Resuscitation; Council on Cardiovascular Nursing; Council on the Kidney in Cardiovascular Disease; Council on Cardiovascular Surgery and Anesthesia, and Interdisciplinary Council on Quality of Care and Outcomes Research. Forecasting the future of cardiovascular disease in the United States: a policy statement from the American Heart Association. Circulation. 2011;123(8):933-944. 
3. Mallika V, Goswami B, Rajappa M. Atherosclerosis pathophysiology and the role of novel risk factors: a clinicobiochemical perspective. Angiology. 2007;58(5):513-522.

4. Fleming RM. The effect of high-, moderate-, and low-fat diets on weight loss and cardiovascular disease risk factors. Prev Cardiol. 2002;5(3): $10-118$.

5. Deanfield JE, Halcox JP, Rabelink TJ. Endothelial function and dysfunction: testing and clinical relevance. Circulation. 2007;115(10): 1285-1295.

6. Tousoulis D, Kampoli AM, Tentolouris C, Papageorgiou N, Stefanadis C. The role of nitric oxide on endothelial function. Curr Vasc Pharmacol. 2012;10(1):4-18.

7. Shah PK. Molecular mechanisms of plaque instability. Curr Opin Lipidol. 2007;18(5):492-499.

8. Calabresi L, Simonelli S, Gomaraschi M, Franceschini G. Genetic lecithin:cholesterol acyltransferase deficiency and cardiovascular disease. Atherosclerosis. 2012;222(2):299-306.

9. Sanz J, Moreno PR, Fuster V. The year in atherothrombosis. J Am Coll Cardiol. 2009;53(15):1326-1337.

10. Falk E. Pathogenesis of atherosclerosis. J Am Coll Cardiol. 2006; 47 (Suppl 8):C7-C12.

11. Stone GW, Maehara A, Lansky AJ, et al. PROSPECT Investigators. A prospective natural-history study of coronary atherosclerosis. $N$ Engl J Med. 2011;364(3):226-235.

12. O'Leary DH, Polak JF, Kronmal RA, Manolio TA, Burke GL, Wolfson SK Jr. Carotid artery intima and media thickness as a risk factor for myocardial infarction and stroke in older adults. Cardiovascular Health Study Collaborative Research Group. N Engl J Med. 1999;340(1): $14-22$.

13. Kavey RE, Daniels SR, Lauer RM, Atkins DL, Hayman LL, Taubert K; American Heart Association. American Heart Association guidelines for primary prevention of atherosclerotic cardiovascular disease beginning in childhood. Circulation. 2003;107(11):1562-1566.

14. Greenland P, Alpert JS, Beller GA, et al. American College of Cardiology Foundation; American Heart Association. 2010 ACCF/ AHA guideline for assessment of cardiovascular risk in asymptomatic adults: a report of the American College of Cardiology Foundation/ American Heart Association Task Force on Practice Guidelines. $J$ Am Coll Cardiol. 2010;56(25):e50-e103.

15. Gidding SS, Lichtenstein AH, Faith MS, et al. Implementing American Heart Association pediatric and adult nutrition guidelines: a scientific statement from the American Heart Association Nutrition Committee of the Council on Nutrition, Physical Activity and Metabolism, Council on Cardiovascular Disease in the Young, Council on Arteriosclerosis, Thrombosis and Vascular Biology, Council on Cardiovascular Nursing, Council on Epidemiology and Prevention, and Council for High Blood Pressure Research. Circulation. 2009;119(8):1161-1175.

16. Zeb I, Budoff MJ. MESA: The NIH-sponsored study that validates atherosclerosis imaging for primary prevention. Curr Atheroscler Rep. 2011;13(5):353-358

17. Naghavi M, Falk E, Hecht HS, et al. SHAPE Task Force. From vulnerable plaque to vulnerable patient - Part III: Executive summary of the Screening for Heart Attack Prevention and Education (SHAPE) Task Force report. Am J Cardiol. 2006;98(2A):2H-15H.

18. Aguilar-Shea AL, Gallardo-Mayo C, Garrido-Elustondo S, Calvo-Manuel E, Zamorano-Gómez JL. Carotid intima-media thickness as a screening tool in cardiovascular primary prevention. Eur J Clin Invest. 2011;41(5):521-526.

19. Hecht HS. The role of atherosclerosis imaging in redefining normal and abnormal cholesterol values, and risk reduction in primary prevention statin trials. Curr Atheroscler Rep. 2011;13(5):422-430.

20. Lenfant F, Trémollières F, Gourdy P, Arnal JF. Timing of the vascular actions of estrogens in experimental and human studies: why protective early, and not when delayed? Maturitas. 2011;68(2):165-173.

21. Deniset JF, Pierce GN. Possibilities for therapeutic interventions in disrupting Chlamydophila pneumoniae involvement in atherosclerosis. Fundam Clin Pharmacol. 2010;24(5):607-617.
22. Pashkow FJ. Oxidative Stress and Inflammation in Heart Disease: Do Antioxidants Have a Role in Treatment and/or Prevention? Int J Inflam. 2011;2011:514623.

23. Reis JP, Loria CM, Steffen LM, et al. Coffee, decaffeinated coffee, caffeine, and tea consumption in young adulthood and atherosclerosis later in life: the CARDIA study. Arterioscler Thromb Vasc Biol. 2010;30(10): 2059-2066.

24. Muntendam P, McCall C, Sanz J, Falk E, Fuster V; High-Risk Plaque Initiative. The BioImage Study: novel approaches to risk assessment in the primary prevention of atherosclerotic cardiovascular disease - study design and objectives. Am Heart J. 2010;160(1):49-57.

25. Dauchet L, Amouyel P, Dallongeville J. Fruits, vegetables and coronary heart disease. Nat Rev Cardiol. 2009;6(9):599-608.

26. Jabłecka A, Bogdański P, Balcer N, Cieślewicz A, Skołuda A, Musialik K. The effect of oral L-arginine supplementation on fasting glucose, $\mathrm{HbA}_{1 \mathrm{c}}$, nitric oxide and total antioxidant status in diabetic patients with atherosclerotic peripheral arterial disease of lower extremities. Eur Rev Med Pharmacol Sci. 2012;16(3): 342-350.

27. Szostak J, Laurant P. The forgotten face of regular physical exercise: a "natural" anti-atherogenic activity. Clin Sci (Lond). 2011;121(3): 91-106.

28. Ahmed HM, Blaha MJ, Nasir K, Rivera JJ, Blumenthal RS. Effects of physical activity on cardiovascular disease. Am J Cardiol. 2012;109(2): 288-295.

29. Ford MA, Allison TG, Lerman A. New approaches to the concept of primary prevention of atherosclerosis. Curr Treat Options Cardiovasc Med. 2008;10(1):73-82.

30. Smith SC Jr, Benjamin EJ, Bonow RO et al. AHA/ACCF Secondary Prevention and Risk Reduction Therapy for Patients with Coronary and other Atherosclerotic Vascular Disease: 2011 update: a guideline from the American Heart Association and American College of Cardiology Foundation. Circulation. 2011 Nov 29;124(22):2458-2473.

31. Smith SC Jr, Benjamin EJ, Bonow RO, et al; World Heart Federation and the Preventive Cardiovascular Nurses Association. AHA/ACCF Secondary Prevention and Risk Reduction Therapy for Patients with Coronary and other Atherosclerotic Vascular Disease: 2011 update: a guideline from the American Heart Association and American College of Cardiology Foundation. Circulation. 2011;124(22): 2458-2473.

32. Lichtman JH, Bigger JT Jr, Blumenthal JA, et al. American Heart Association Prevention Committee of the Council on Cardiovascular Nursing; American Heart Association Council on Clinical Cardiology; American Heart Association Council on Epidemiology and Prevention; American Heart Association Interdisciplinary Council on Quality of Care and Outcomes Research; American Psychiatric Association. Depression and coronary heart disease: recommendations for screening, referral, and treatment: a science advisory from the American Heart Association Prevention Committee of the Council on Cardiovascular Nursing, Council on Clinical Cardiology, Council on Epidemiology and Prevention, and Interdisciplinary Council on Quality of Care and Outcomes Research: endorsed by the American Psychiatric Association. Circulation. 2008;118(17): 1768-1775.

33. Leon AS, Franklin BA, Costa F, et al; American Heart Association; Council on Clinical Cardiology (Subcommittee on Exercise, Cardiac Rehabilitation, and Prevention); Council on Nutrition, Physical Activity, and Metabolism (Subcommittee on Physical Activity); American association of Cardiovascular and Pulmonary Rehabilitation. Cardiac rehabilitation and secondary prevention of coronary heart disease: an American Heart Association scientific statement from the Council on Clinical Cardiology (Subcommittee on Exercise, Cardiac Rehabilitation, and Prevention) and the Council on Nutrition, Physical Activity, and Metabolism (Subcommittee on Physical Activity), in collaboration with the American association of Cardiovascular and Pulmonary Rehabilitation. Circulation. 2005;111(3): 369-376. 
34. Brott TG, Halperin JL, Abbara S, et al; American College of Cardiology Foundation; American Stroke Association; American Association of Neurological Surgeons; American College of Radiology; American Society of Neuroradiology; Congress of Neurological Surgeons; Society of Atherosclerosis Imaging and Prevention; Society for Cardiovascular Angiography and Interventions; Society of Interventional Radiology; Society of NeuroInterventional Surgery; Society for Vascular Medicine; Society for Vascular Surgery. 2011 ASA/ACCF/AHA/AANN/ AANS/ACR/ASNR/CNS/SAIP/SCAI/SIR/SNIS/SVM/SVS guideline on the management of patients with extracranial carotid and vertebral artery disease: executive summary. A report of the American College of Cardiology Foundation/American Heart Association Task Force on Practice Guidelines, and the American Stroke Association, American Association of Neuroscience Nurses, American Association of Neurological Surgeons, American College of Radiology, American Society of Neuroradiology, Congress of Neurological Surgeons, Society of Atherosclerosis Imaging and Prevention, Society for Cardiovascular Angiography and Interventions, Society of Interventional Radiology, Society of NeuroInterventional Surgery, Society for Vascular Medicine, and Society for Vascular Surgery. Circulation. 2011;124(4):489-532.

35. Wallentin L, Becker RC, Budaj A, et al; PLATO Investigators, Freij A, Thorsén M. Ticagrelor versus clopidogrel in patients with acute coronary syndromes. $N$ Engl J Med. 2009;361(11):1045-1057.

36. Wiviott SD, Braunwald E, McCabe CH, et al; TRITON-TIMI 38 Investigators. Prasugrel versus clopidogrel in patients with acute coronary syndromes. $N$ Engl J Med. 2007;357(20):2001-2015.

37. Gerstein HC, Ratner RE, Cannon CP, et al; APPROACH Study Group. Effect of rosiglitazone on progression of coronary atherosclerosis in patients with type 2 diabetes mellitus and coronary artery disease: the assessment on the prevention of progression by rosiglitazone on atherosclerosis in diabetes patients with cardiovascular history trial. Circulation. 2010;121(10):1176-1187.

38. García-García HM, Garg S, Brugaletta S, et al; APPROACH study group. Evaluation of in-stent restenosis in the APPROACH trial (assessment on the prevention of progression by Rosiglitazone on atherosclerosis in diabetes patients with cardiovascular history). Int $J$ Cardiovasc Imaging. 2012;28(3):455-465.

39. Francis AA, Pierce GN. An integrated approach for the mechanisms responsible for atherosclerotic plaque regression. Exp Clin Cardiol. 2011;16(3):77-86.

40. Bedi U, Singh M, Singh P, Molnar J, Khosla S, Arora R. Effects of statins on progression of coronary artery disease as measured by intravascular ultrasound. J Clin Hypertens (Greenwich). 2011;13(7): 492-496.

41. Han SH, Chung WJ, Kang WC, et al. Rosuvastatin combined with ramipril significantly reduced atheroma volume by anti-inflammatory mechanism: Comparative analysis with rosuvastatin alone by intravascular ultrasound. Int J Cardiol. 2012;158(2):217-224.

42. Takayama T, Hiro T, Yamagishi M, et al; COSMOS Investigators. Effect of rosuvastatin on coronary atheroma in stable coronary artery disease: multicenter coronary atherosclerosis study measuring effects of rosuvastatin using intravascular ultrasound in Japanese subjects (COSMOS). Circ J. 2009;73(11):2110-2117.

43. Hibi K, Kimura T, Kimura K, et al; JAPAN-ACS Investigators. Clinically evident polyvascular disease and regression of coronary atherosclerosis after intensive statin therapy in patients with acute coronary syndrome: serial intravascular ultrasound from the Japanese assessment of pitavastatin and atorvastatin in acute coronary syndrome (JAPAN-ACS) trial. Atherosclerosis. 2011;219(2):743-749.

44. Arai H, Hiro T, Kimura T, et al; JAPAN-ACS Investigators. More intensive lipid lowering is associated with regression of coronary atherosclerosis in diabetic patients with acute coronary syndrome - sub-analysis of JAPAN-ACS study. J Atheroscler Thromb. 2010;17(10):1096-1107.

45. Hong YJ, Jeong MH, Hachinohe D, et al. Comparison of Effects of rosuvastatin and atorvastatin on plaque regression in Korean patients with untreated intermediate coronary stenosis. Circ J. 2011;75(2): 398-406.
46. Tani S, Nagao K, Anazawa T, et al. Relation of change in apolipoprotein B/apolipoprotein A-I ratio to coronary plaque regression after Pravastatin treatment in patients with coronary artery disease. Am J Cardiol. 2010;105(2):144-148.

47. Kawahara T, Nishikawa M, Furusawa T, Inazu T, Suzuki G. Effect of atorvastatin and etidronate combination therapy on regression of aortic atherosclerotic plaques evaluated by magnetic resonance imaging. J Atheroscler Thromb. 2011;18(5):384-395.

48. Ko YG, Kim BK, Lee BK, et al. SECURE Investigators. Study design and rationale of "Synergistic effect of combination therapy with cilostazol and ProbUcol on plaque stabilization and lesion REgression (SECURE)" study: a double-blind randomised controlled multicenter clinical trial. Trials. 2011;12:10.

49. Kovarnik T, Mintz GS, Skalicka H, et al. Virtual histology evaluation of atherosclerosis regression during atorvastatin and ezetimibe administration: HEAVEN study. Circ J. 2012;76(1):176-183.

50. Migrino RQ, Bowers M, Harmann L, Prost R, LaDisa JF Jr. Carotid plaque regression following 6-month statin therapy assessed by $3 \mathrm{~T}$ cardiovascular magnetic resonance: comparison with ultrasound intima media thickness. J Cardiovasc Magn Reson. 2011;13:37.

51. Nicholls SJ, Ballantyne CM, Barter PJ, et al. Effect of two intensive statin regimens on progression of coronary disease. N Engl J Med. 2011; 365(22):2078-2087.

52. Nicholls SJ, Tuzcu EM, Sipahi I, et al. Statins, high-density lipoprotein cholesterol, and regression of coronary atherosclerosis. JAMA. 2007; 297(5):499-508.

53. Yang HB, Zhao XY, Zhang JY, Du YY, Wang XF. Pioglitazone induces regression and stabilization of coronary atherosclerotic plaques in patients with impaired glucose tolerance. Diabet Med. 2012;29(3): 359-365.

54. Nakayama T, Komiyama N, Yokoyama M, et al. Pioglitazone induces regression of coronary atherosclerotic plaques in patients with type 2 diabetes mellitus or impaired glucose tolerance: a randomized prospective study using intravascular ultrasound. Int J Cardiol. 2010;138(2):157-165.

55. Clementi F, Di Luozzo M, Mango R, et al. Regression and shift in composition of coronary atherosclerotic plaques by pioglitazone: insight from an intravascular ultrasound analysis. J Cardiovasc Med (Hagerstown). 2009;10(3):231-237.

56. Kadoglou NP, Sailer N, Moumtzouoglou A, Kapelouzou A, Gerasimidis T, Liapis CD, et al. Aggressive lipid-lowering is more effective than moderate lipid-lowering treatment in carotid plaque stabilization. J Vasc Surg. 2010;51(1):114-121.

57. Shai I, Spence JD, Schwarzfuchs D, et al; DIRECT Group. Dietary intervention to reverse carotid atherosclerosis. Circulation. 2010;121(10): 1200-1208.

58. Godin B, Sakamoto JH, Serda RE, Grattoni A, Bouamrani A, Ferrari M. Emerging applications of nanomedicine for therapy and diagnosis of cardiovascular diseases. Trends Pharmacol Sci. 2010;31(5):199-205.

59. Lobatto ME, Fuster V, Fayad ZA, Mulder WJ. Perspectives and opportunities for nanomedicine in the management of atherosclerosis. Nat Rev Drug Discov. 2011;10(11):835-852.

60. O'Neil CP, van der Vlies AJ, Velluto D, et al. Extracellular matrix binding mixed micelles for drug delivery applications. $J$ Control Release. 2009;137(2):146-151.

61. Chan JM, Zhang L, Tong R, et al. Spatiotemporal controlled delivery of nanoparticles to injured vasculature. Proc Natl Acad Sci U S A. 2010; 107(5):2213-2218.

62. Gordon T, Castelli WP, Hjortland MC, Kannel WB, Dawber TR. High density lipoprotein as a protective factor against coronary heart disease. The Framingham Study. Am J Med. 1977;62(5):707-714.

63. Sharrett AR, Ballantyne CM, Coady SA, et al. Coronary heart disease prediction from lipoprotein cholesterol levels, triglycerides, lipoprotein(a), apolipoproteins A-I and B, and HDL density subfractions: The Atherosclerosis Risk in Communities (ARIC) Study. Circulation. 2001;104(10):1108-1113.

64. Forrester JS, Shah PK. Emerging strategies for increasing high-density lipoprotein. Am J Cardiol. 2006;98(11):1542-1549. 
65. Fayad ZA, Mani V, Woodward M, et al; dal-PLAQUE Investigators. Safety and efficacy of dalcetrapib on atherosclerotic disease using novel non-invasive multimodality imaging (dal-PLAQUE): a randomised clinical trial. Lancet. 2011;378(9802):1547-1559.

66. Boettcher MF, Heinig R, Schmeck C, et al. Single dose pharmacokinetics, pharmacodynamics, tolerability and safety of BAY 60-5521, a potent inhibitor of cholesteryl ester transfer protein. $\mathrm{Br} \mathrm{J} \mathrm{Clin}$ Pharmacol. 2012;73(2):210-218.

67. Gutstein DE, Krishna R, Johns D, et al. Anacetrapib, a novel CETP inhibitor: pursuing a new approach to cardiovascular risk reduction. Clin Pharmacol Ther. 2012;91(1):109-122.

68. Cannon CP, Dansky HM, Davidson M, et al; DEFINE investigators. Design of the DEFINE trial: determining the EFficacy and tolerability of CETP INhibition with AnacEtrapib. Am Heart J. 2009;158(4): 513-519.e3.

69. Chen F, Maccubbin D, Yan L, et al. Lipid-altering efficacy and safety profile of co-administered extended release niacin/laropiprant and simvastatin versus atorvastatin in patients with mixed hyperlipidemia. Int $J$ Cardiol. 2012. Epub Feb 3.

70. Taylor AJ, Sullenberger LE, Lee HJ, Lee JK, Grace KA. Arterial Biology for the Investigation of the Treatment Effects of Reducing Cholesterol (ARBITER) 2: a double-blind, placebo-controlled study of extendedrelease niacin on atherosclerosis progression in secondary prevention patients treated with statins. Circulation. 2004;110(23):3512-3517.

71. Villines TC, Stanek EJ, Devine PJ, et al. The ARBITER 6-HALTS Trial (Arterial Biology for the Investigation of the Treatment Effects of Reducing Cholesterol 6-HDL and LDL Treatment Strategies in Atherosclerosis): final results and the impact of medication adherence, dose, and treatment duration. J Am Coll Cardiol. 2010;55(24):2721-2726.

72. Yadav R, France M, Younis N, et al. Extended-release niacin with laropiprant: a review on efficacy, clinical effectiveness and safety. Expert Opin Pharmacother. 2012;13(9):1345-1362.

73. University of Oxford. Treatment of HDL to reduce the incidence of vascular events HPS2-THRIVE. In: ClinicalTrials.gov [website on the Internet]. Bethseda, MD: US National Library of Medicine; 2007 [updated July 19, 2010]. Available from: http://www.clinicaltrials.gov/ ct2/show/NCT00461630. NLM identifier: NCT00461630. Accessed July 5, 2012

74. University of Washington. Carotid plaque characteristics by MRI in AIM-HIGH (Carotid MRI Substudy). In: ClinicalTrials.gov [website on the Internet]. Bethseda, MD: US National Library of Medicine; 2010 [updated June 19, 2012]. Available from: http://www.clinicaltrials.gov/ ct2/show/NCT01178320. NLM identifier: NCT01178320. Accessed July 5, 2012
75. National Heart, Lung, and Blood Institute. Plaque inflammation and dysfunctional hdl cholesterol in participants receiving niacin and statins in the AIM-HIGH Study (The HDL Proteomics Study). In: ClinicalTrials.gov [website on the Internet]. Bethseda, MD: US National Library of Medicine; 2009 [updated February 23, 2012]. Available from: http://www.clinicaltrials.gov/ct2/show/NCT00880178. NLM identifier: NCT00880178. Accessed July 5, 2012.

76. Buse JB, Rubin CJ, Frederich R, et al. Muraglitazar, a dual (alpha/gamma) PPAR activator: a randomized, double-blind, placebo-controlled, 24-week monotherapy trial in adult patients with type 2 diabetes. Clin Ther. 2005;27(8):1181-1195.

77. Goldstein BJ, Rosenstock J, Anzalone D, Tou C, Ohman KP. Effect of tesaglitazar, a dual PPAR alpha/gamma agonist, on glucose and lipid abnormalities in patients with type 2 diabetes: a 12-week dose-ranging trial. Curr Med Res Opin. 2006;22(12):2575-2590.

78. Saad MF, Greco S, Osei K, et al; Ragaglitazar Dose-Ranging Study Group. Ragaglitazar improves glycemic control and lipid profile in type 2 diabetic subjects: a 12-week, double-blind, placebo-controlled dose-ranging study with an open pioglitazone arm. Diabetes Care. 2004;27(6):1324-1329.

79. Tardif JC, Grégoire J, L'Allier PL, et al; Effect of rHDL on Atherosclerosis-Safety and Efficacy (ERASE) Investigators. Effects of reconstituted high-density lipoprotein infusions on coronary atherosclerosis: a randomized controlled trial. JAMA. 2007;97(15):1675-1682.

80. Waksman R, Torguson R, Kent KM, et al. A first-in-man, randomized, placebo-controlled study to evaluate the safety and feasibility of autologous delipidated high-density lipoprotein plasma infusions in patients with acute coronary syndrome. J Am Coll Cardiol. 2010;55(24): 2727-2735.

81. Cerenis Therapeutics, SA. Effect of CER-001 on atherosclerosis in acute coronary syndrome (ACS) patients - efficacy and safety: the CHI SQUARE Trial. In: ClinicalTrials.gov [website on the Internet]. Bethseda, MD: US National Library of Medicine; 2010 [updated June 14, 2012]. Available from: http://www.clinicaltrials.gov/ct2/ show/NCT01201837. NLM identifier: NCT01201837. Accessed July 5, 2012.

82. Resverlogix Corp. National Institute of Health ApoA-I synthesis stimulation and intravascular ultrasound for coronary atheroma regression evaluation. In: ClinicalTrials.gov [website on the Internet]. Bethseda, MD: US National Library of Medicine; 2010 [updated June 27, 2012]. Available from: http://www.clinicaltrials.gov/ct2/show/NCT01067820. NLM identifier: NCT01067820. Accessed July 5, 2012.
Vascular Health and Risk Management

\section{Publish your work in this journal}

Vascular Health and Risk Management is an international, peerreviewed journal of therapeutics and risk management, focusing on concise rapid reporting of clinical studies on the processes involved in the maintenance of vascular health; the monitoring, prevention and treatment of vascular disease and its sequelae; and the involvement of

\section{Dovepress}

metabolic disorders, particularly diabetes. This journal is indexed on PubMed Central and MedLine. The manuscript management system is completely online and includes a very quick and fair peer-review system, which is all easy to use. Visit http://www.dovepress.com/ testimonials.php to read real quotes from published authors. 